Objectives In endometrial cancer few randomized controlled trials were conducted to assess the role of different settings of follow-up in improving overall survival. The TOTEM study (NCT00916708) was planned to compare an intensive (INT) vs minimalist (MIN) 5-year follow-up regimen in endometrial cancer patients in terms of overall survival (OS).

Methods Patients surgically treated for endometrial cancer, were stratified by center and in low (LoR) or high (HiR) risk of recurrence and then randomized to INT or MIN hospitalbased follow-up regimens. The aim of the study was to demonstrate an improvement from $75 \%$ to $80 \%$ (expected hazard ratio, $\mathrm{HR}=0.78$ ) of the 5 -year OS with the INT regimen. Secondary objectives were to compare relapse free survival (RFS) and health-related quality of life (HRQL).

Results 1884 patients were randomized in 42 centers between 2008 and 2018, and 1847 patients were available for the final analysis. After a median follow-up of 66 months, the 5-year OS was $91.3 \%, 90.6 \%$ in the INT and $91.9 \%$ in the MIN arms, respectively $(\mathrm{HR}=1.12,95 \% \mathrm{CI} \quad 0.85-1.48, \mathrm{p}=0.429)$. Comparing the INT vs MIN arms, the 5-year OS were $94.1 \%$ and $96.8 \%(\mathrm{HR}=1.48,0.92-2.37, \mathrm{p}=0.104)$ in the LoR and $85.3 \%$ and $84.7 \% \quad(\mathrm{HR}=0.96,0.68-1.36, \mathrm{p}=0.814)$ in the HiR group. The two arms did not show differences in terms of RFS and HRQL.

Conclusions Intensive follow-up in endometrial cancer treated patients did not improve OS, even in HiR patients, nor influenced health-related quality of life. Frequent routine use of imaging and laboratory exams in these patients should be discouraged.

\section{5/\#190 WHERE THERE IS SMOKE, THERE IS FIRE: UNDERSTANDING THE IMPLICATIONS OF POSITIVE SENTINEL LYMPH NODES IN ENDOMETRIAL CANCER}

\begin{abstract}
${ }^{1,2} \mathrm{G}$ Dinoi ${ }^{*},{ }^{2} \mathrm{~K}$ Ghoniem, ${ }^{3} \mathrm{Y}$ Huang, ${ }^{2} \mathrm{~V}$ Zanfagnin, ${ }^{2} \mathrm{C}$ Langstraat, ${ }^{2} \mathrm{G}$ Glaser, ${ }^{4} \mathrm{~A}$ Weaver, ${ }^{4} \mathrm{M}$ Mcgree, ${ }^{5} \mathrm{~F}$ Fanfani, ${ }^{5} \mathrm{G}$ Scambia, ${ }^{2} \mathrm{~A}$ Mariani. ${ }^{1}$ Fondazione Policlinico A. Gemelli, IRCCS, Gynaecologic Oncology, Rome, Italy; ${ }^{2}$ Mayo Clinic, Division of Gynaecologic Surgery, Department of Obstetrics and Gynaecology, Rochester, USA; ${ }^{3}$ Mayo Clinic, Division of Anatomic Pathology, Rochester, USA; ${ }^{4}$ Division of Biomedical Statistics and Informatics, Department of Health Sciences Research, Rochester, USA; ${ }^{5}$ Università Cattolica del Sacro Cuore, Department of Woman and Child Health and Public Health, Woman Health Area, Fondazione Policlinico Universitario A. Gemelli Irccs, Rome, Italy
\end{abstract}

\subsection{6/ijgc-2021-IGCS.5}

Objectives The objective of this study is to identify clinicopathologic characteristics associated with non-sentinel lymph node (SLN) metastasis and non-vaginal recurrences in patients with SLN-positive endometrial cancer (EC).

Methods Consecutive patients with surgically staged EC and at least one positive SLN were included. SLNs were ultra-staged. Positive SLNs were reviewed and patients classified according to the size of the largest SLN metastasis.

Results 103 patients (36 isolated tumor cells (ITC), 27 micrometastasis, 40 macrometastasis) were included. Multiple positive SLNs were observed in $38.8 \%$ of patients. Size of SLN metastasis (adjusted OR (aOR) 3.0 for macrometastasis vs ITC, 95\%CI 1.1-8.1), and age (aOR 1.8 per 10-year increase, $95 \%$ CI 1.1-3.0) were independent predictors of multiple positive SLNs. Extracapsular compared to intracapsular invasion of
Abstract 0005/\#190 Table 1

\begin{tabular}{|l|l|l|}
\hline Characteristic & $\begin{array}{l}\text { Adjusted } \\
\text { HR }(95 \% \mathrm{CI})\end{array}$ & $\mathrm{P}$ \\
\hline Histology & & 0.004 \\
\hline Non-endometrioid & $5.09(1.66,15.61)$ & \\
\hline Endometrioid & Reference & \\
\hline Cervical stromal invasion & & 0.002 \\
\hline No & Reference & \\
\hline Yes & $6.89(2.04,23.23)$ & \\
\hline Size of SLN metastasis & & 0.04 \\
\hline ITC/micrometastasis & Reference & \\
\hline Macrometastasis & $3.41(1.05,11.09)$ & \\
\hline
\end{tabular}

the SLN metastasis was significantly associated with multiple positive SLNs at univariate analysis $(71.4 \%$ vs. $33.7 \%$, $\mathrm{p}=0.008)$. Forty-seven percent $(18 / 38)$ of patients who underwent completion pelvic lymphadenectomy, had additional positive lymph nodes. This was associated with increased size of SLN metastasis $(0 / 8,5 / 10$, and 13/20 in ITC, micro- and macrometastasis, respectively, $\mathrm{p}=0.004)$. SLN macrometastasis (adjusted HR (aHR) 3.4, 95\%CI 1.1-11.0), non-endometrioid histology (aHR 5.7, 95\%CI 1.9-17.3), and cervical stromal invasion (aHR 9.4, 95\%CI 2.9-30.4) were independent predictors of non-vaginal recurrence (table 1).

Conclusions Size and location of SLN metastasis can predict an increased risk of multiple positive SLNs, non-SLN positive nodes, and non-vaginal recurrence in SLN positive EC patients. These factors should be assessed when considering adjuvant treatment in these high-risk patients.

\section{6/\#340 MINIMALLY INVASIVE SURGERY IN ADVANCED ENDOMETRIAL CARCINOMA IS ASSOCIATED WITH AN INCREASED RISK FOR LOCAL RECURRENCE}

${ }^{1} \mathrm{~L}$ Kogan*, ${ }^{2} \mathrm{G}$ Levin, ${ }^{3} \mathrm{~L}$ Helpman, ${ }^{4} \mathrm{R}$ Eitan, ${ }^{5} \mathrm{Z}$ Vaknin, ${ }^{6} \mathrm{O}$ Lavie, ${ }^{7} \mathrm{~A}$ Ben Arie, ${ }^{8} \mathrm{~A}$ Amit, ${ }^{9} \mathrm{~T}$ Levy, ${ }^{10} \mathrm{~A}$ Namazov, ${ }^{11}$ I Ben Shachar, ${ }^{12}$ I Atlas, ${ }^{13}$ I Bruchim, ${ }^{1} \mathrm{~B}$ Brandt, ${ }^{14} \mathrm{O}$ Gemer. ${ }^{1}$ Hadassah, Gynecologic Oncology, Jerusalem, Israel; ${ }^{2}$ hadassah medical center, Gynecologic Oncology, jerusalem, Israel; ${ }^{3}$ Cheba medical center, Gynecologic Oncology, Ramat Gan, Israel; ${ }^{4}$ Rabin Medical Center, Gynecology, Petah Tikva, Israel; ${ }^{5}$ Assaf Haroffe Medical Center, Sackler School of Medicine, Gynecology, Zrifin, Israel; ${ }^{6}$ Carmel Medical Center, Obstetrics and Gynecology, Haifa, Israel; 72 2. Kaplan Medical Center, Hebrew University, Gynecology, Rehovot, Israel; ${ }^{8}$ Rambam, Gymecologic Oncology, Haifa, Israel; ${ }^{9} 8$. Wolfson Medical Center, Holon, Sackler Faculty of Medicine, Tel Aviv University, Gynecology, Tel Aviv, Israel; ${ }^{10}$ Ahmet, Gynecology, Ashkelon, Israel; ${ }^{11}$ Zlv, Gynecologic Oncology, Zefat, Israel; ${ }^{12}$ Poriah, Gynecologic Oncology, Tiberia, Israel; ${ }^{13}$ Hillel Yafe, Gynecology, Hadera, Israel; ${ }^{14}$ Barzilai Medical Center, Gynecology, Ashkelon, Israel

\subsection{6/ijgc-2021-IGCS.6}

Objectives To compare oncological outcomes of women with stage II -IIIc endometrial cancer (EC) who underwent minimally invasive surgery (MIS) versus laparotomy.

Methods A retrospective cohort study in an academic multicenter setting. Consecutive women with EC treated at 11 Israeli institutions between 2002 and 2017 were recorded in an assimilated database with a median follow-up of 52 months (range 12-120 months). Women with stage II -IIIc were stratified into groups by intentional route of surgery; MIS vs. laparotomy. Clinical, pathological and outcome data were compared. 